Génét. Sél. Evol., 1988, 20 (2), 181-198

\title{
Distribution spatiale des génotypes dans une population de chêne vert (Quercus ilex L.), flux génique et régime de reproduction
}

\author{
Assia YACINE et Roselyne LUMARET \\ Centre National de la Recherche Scientifique, \\ Unité de Biologie des Populations et des Peuplements, \\ Centre L. Emberger, route de Mende, \\ BP 5051, 34033 Montpellier Cedex, France
}

\begin{abstract}
Résumé
L'analyse de la distribution spatiale des génotypes dans une station de chêne vert (Quercus ilex L.) à l'aide de trois systèmes enzymatiques (PGI-1, IDH-1 et ADH-1) montre l'absence d'un effet d'agrégation spatiale des diverses classes génotypiques quel que soit le locus considéré. L'indice de fixation Fis de Wright, à l'échelle de la station, n'est pas significativement différent de zéro (Fis $=0,02)$ et indique une situation proche de l'équilibre de Hardy-Weinberg. Les fréquences alléliques dans le pollen ayant participé à la production des descendances respectives de neuf arbres issus de cette même station sont significativement différentes (au risque de $5 \%$ ) d'une mère à l'autre, même quand celles-ci sont spatialement très proches. Les arbres ne sont pas nécessairement pollinisés par les individus les plus proches mais par ceux qui leur sont à la fois phénologiquement synchrones et qui ont prioritairement investi dans la fonction mâle.
\end{abstract}

Mots clés: Quercus ilex L., structure génétique, régime de reproduction, allozyme.

\section{Summary}

Spatial distribution of genotypes in a population of holm oak (Quercus ilex L.), gene flow and mating system

Spatial distribution of genotypes in holm oak (Quercus ilex L.) was analyzed within a site using three allozyme systems (PGI-1, IDH-1, ADH-1) as markers. No aggregation effect of the genotypic classes could be pointed out at any locus. Wright's fixation index calculated over all the individuals of the site was not significantly different from zero $(F i s=0.02)$, indicating a situation close to the Hardy-Weinberg equilibrium expectation. Allele frequencies in the effective pollen responsible for the observed progenies of nine open-pollinated individual trees of the site were significantly different $(P<0.05)$ even when the parents were very close. Finally, the results clearly show that the trees are not necessarily pollinated by their neighbours, but rather by those individuals that are both synchronous phenologically and that invest primarily in the male function.

Key words : Quercus ilex L., genetic structure, mating system, allozyme. 


\section{Introduction}

La relation génétique entre deux générations d'une espèce végétale à reproduction sexuée dépend de conditions intrinsèques à l'espèce comme, par exemple, l'existence ou non d'un système d'auto-incompatibilité, de l'intensité du flux pollinique et de son mode de dispersion et de la densité des individus constituant la population parentale : dans une population à faible densité, chaque individu reçoit un pool pollinique plus diversifié que lorsqu'il existe une forte densité (Cheliak et al., 1985). Dans ce dernier cas en effet, une anisotropie correspondant à un effet de masquage peut se produire (BARAdat et al., 1984). Lorsque l'espèce considérée se caractérise par une variabilité phénologique entre individus, le régime de reproduction dépend aussi de la localisation des individus dans la station quel que soit leur degré d'apparentement.

Les études expérimentales portant à la fois sur la structure génétique d'une population et sur celle de descendants issus de celle-ci ont été réalisées pour certaines espèces telles que Pinus pinaster (Baradat et al., 1984), Picea abies (Brunel \& Rodolphe, 1985), Sorghum bicolor (Ellstrand \& Foster, 1983), Cinosurus cristatus (Ennos, 1985) et Pinus taeda (Roberds \& Conkle, 1984). Pour certaines d'entre elles, on a montré l'impact de la structure spatiale sur les taux d'allofécondation. Ainsi, ENNOS \& GlEGG (1982) démontrent que l'agrégation spatiale des génotypes d'une population d'Ipomea purpurea engendre une diminution du taux d'allofécondation apparent. Nous traitons ici le cas du chêne vert (Quercus ilex L.). Cette espèce est monoïque, à longue génération et anémophile. Elle présente en outre une grande variabilité phénologique à la fois entre stations et au sein d'une même station (Du Merle, 1983). L'impact de ce dernier paramètre sur la restriction du flux pollinique efficace a été démontré aussi bien au niveau théorique (STAM, 1983) qu'expérimental (McNeilly \& Antonovics, 1968). L'effet de la phénologie sur la réduction de l'effectif génétique a été également mis en évidence dans le cas de Pseudotsuga menziesii (EL KASSABY et al., 1984). A l'aide de marqueurs enzymatiques, nous avons analysé d'une part, l'organisation spatiale des génotypes dans une station de la région montpelliéraine (Puéchabon) et d'autre part, les distributions alléliques dans les descendances d'un certain nombre d'individus fécondés in situ.

\section{Matériel et méthodes}

\section{A. La station}

\section{Matériel}

Dans la station de Puéchabon, une parcelle rectangulaire de $490 \mathrm{~m}^{2}$ $(20 \mathrm{~m} \times 24,50 \mathrm{~m})$ a été délimitée dans un peuplement de plusieurs centaines d'hectares et la position respective de tous les arbres y a été cartographiée. Sur ses côtés est et sud, la parcelle est bordée sur $5 \mathrm{~m}$ de large environ de chênes verts eux-mêmes isolés du reste du peuplement par des chemins ( $4 \mathrm{~m}$ de large environ). Sur ses côtés nord et ouest, la parcelle forme un couvert continu avec le reste du peuplement. Le prélèvement du matériel constitué de rameaux feuillés et destiné à l'analyse enzymatique a été 
effectué sur tous les individus de la parcelle. Dans le cas du chêne vert, la notion d'individu n'est pas toujours claire, lorsque plusieurs brins émergent d'une même souche. Dans les sept cas où la question se posait, le matériel a été récolté séparément par brin sur les cépées.

L'analyse de trois systèmes enzymatiques (phosphogluco-isomérase PGI, isocitratedéshydrogénase IDH et alcool-déshydrogénase $\mathrm{ADH}$ ) a été réalisée pour chaque échantillon. Les techniques utilisées ainsi que le déterminisme génétique de ces marqueurs sont décrits par ailleurs (Yacine, 1987 ; YaCine \& LumareT, 1988).

\section{Analyse de la structure génétique}

Les marqueurs enzymatiques ont été utilisés pour tenter de déterminer le nombre d'individus distincts : on a considéré que les brins d'une même cépée présentant le même génotype pour les trois locus appartenaient au même individu. Cette considération sous-entend que l'on admet que l'espèce est essentiellement allogame. Les premières autofécondations forcées réalisées sur 37 arbres n'ont pas abouti à la production de glands (YACINE, 1987).

A partir des génotypes des 158 individus ainsi identifiées, on a calculé les indices de fixation Fis et Fst (WrIGHT, 1951 ; KIRBY, 1975 ; NeI, 1977) en utilisant diverses échelles de subdivision. La première trame correspond à une subdivision en carrés d'un demi-mètre de côté, la maille 2 , à des carrés d'1 $\mathbf{m}$ de côté, la maille 3 à des carrés d' $1,50 \mathrm{~m}$ de côté ; on ajoute ainsi $0,50 \mathrm{~m}$ aux côtés des carrés pour obtenir l'échelle suivante de subdivision et ce, jusqu'à la maille qui englobe la totalité de la parcelle.

Les tailles de maille pour lesquelles d'une part la variation des effectifs par carré marque un point d'inflexion et descend en dessous de 6 (valeur arbitrairement retenue qui correspond à la plupart des cas observés) et d'autre part l'effectif par maille reste suffisant (supérieur à 5) sont particulièrement intéressantes car elles correspondent à une distribution spatiale aussi homogène que possible entre les différentes mailles.

Pour le calcul des indices de fixation, n'ont été pris en compte que les carrés présentant plus d'un allèle pour l'ensemble des génotypes qui y étaient inscrits.

Fis estime la corrélation intra-individuelle entre allèles identiques à l'intérieur des groupes d'individus ou " populations " composant l'espèce et représente l'écart à la panmixie. Il varie entre -1 quand les allèles identiques ne sont jamais associés et +1 lorsqu'ils le sont toujours. Quand l'association se fait au hasard, Fis $=0$.

Dans ce travail, la formule corrigée de Kirby (1975) qui tient compte des fluctuations d'effectifs des échantillons a été utilisée.

$$
F i s_{i l u}=1-\frac{H_{i t u}}{2 P_{i t u}\left(1-P_{i t h}\right)}+\frac{1}{2 N_{i l}-1}
$$

où $H_{i l u}$ est la fréquence observée en hétérozygotes dans la population $i$ au locus $l$ et pour l'allèle $u ; P_{i l u}$ est la fréquence estimée de cet allèle et $N_{i l}$ l'effectif de l'échantillon pour le locus $l$ dans la population $i$. Pour obtenir le Fis moyen pour un locus, on a utilisé une moyenne pondérée selon les fréquences alléliques :

$$
F i s_{i l}=\frac{\Sigma_{u} P_{i l u}-\left(1-P_{i t u}\right) F i s_{i t u}}{\Sigma_{u} P_{i l u}\left(1-P_{i t u}\right)}
$$


Fst représente la corrélation entre allèles identiques dans un groupe par rapport à l'ensemble des groupes ; il estime donc la différentiation intergroupe.

$$
F s t=\frac{\operatorname{Var}\left(P_{i}\right)}{\overline{\mathrm{P}}(1-\overline{\mathrm{P}})}
$$

où $\bar{P}$ est la fréquence allélique moyenne dans l'ensemble des groupes et Var $\left(P_{i}\right)$ est la variance des fréquences alléliques dans chaque groupe.

La formule utilisée a été corrigée en fonction de l'effectif des échantillons. Les estimations pour chaque allèle sont combinées pour le locus sous forme d'une moyenne pondérée et l'on fait de même pour obtenir la valeur correspondant à l'ensemble des locus.

\section{B. Les descendances}

\section{Matériel}

La totalité des glands issus de fécondation libre et produits par 9 arbres disséminés dans la parcelle ont été récoltés (fig. 1, 2 et 3). Ces arbres étaient relativement de petite taille et la plupart des glands se trouvaient au sommet de la couronne foliaire. La quantité globale récoltée correspond approximativement à $70 \%$ de la production totale de la parcelle pour l'année de récolte. Les glands ont été mis à germer selon les méthodes préconisées par AISSA (1981). L'analyse des trois systèmes enzymatiques, (PGI, IDH et $\mathrm{ADH}$ ) a été réalisée sur les 492 plantules âgées d'un mois et demi et cultivées en serre de façon homogène, les conditions d'humidité ayant été entretenues par des arrosages réguliers.

\section{Méthodes de calcul}

Un test d'homogénéité du $\chi^{2}$ a été utilisé pour comparer, entre les descendances issues des mères qui présentaient des génotypes identiques entre elles, les distributions alléliques à chaque locus dans le pollen ayant contribué à la formation des descendants.

Une estimation des taux d'allofécondation a été réalisée pour chaque mère à partir de la méthode de SHaw et al. (1981) puis, pour l'ensemble des mères, selon la méthode de Green et al. (1980). Selon la méthode de Shaw et collaborateurs, l'estimateur « multilocus» du taux d'allofécondation $t_{m}$ est obtenu à partir de la fréquence des descendants issus avec certitude d'allofécondations (ils possèdent au moins un allèle absent chez leur mère) et de la probabilité de formation de descendants par allofécondation, ces derniers ne pouvant être détectés directement :

$t_{m}=\frac{n}{N(1-\alpha)}$ où $n$ est le nombre de descendants issus d'allofécondations discernables, $N$ est l'effectif de la descendance et $\alpha$ la probabilité de non-identification d'une autofécondation alors que celle-ci a lieu.

$$
\alpha=\prod_{k=1}^{m}\left[\Sigma_{i} \Sigma_{j} f_{k}\left(A_{i} A_{j}\right)\left(P_{i k}+P_{j k}\right)+\Sigma_{i} f_{k}\left(A_{i} A_{i}\right) P_{i k}\right]
$$

où $f_{k}\left(A_{i} A_{i}\right)$ et $f_{k}\left(A_{i} A_{j}\right)$ sont les fréquences de génotypes $A_{i} A_{i}$ (homozygote) et $A_{i} A_{j}$ (héterrozygote) au $k^{e}$ locus pondérées par le nombre de descendants que chaque génotype produit, pour $m$ locus. $P_{i k}$ et $P_{i k}$ sont les fréquences alléliques dans le pollen au $k^{\mathrm{e}}$ locus. 
La variance de $t_{m}$ est :

$$
\operatorname{Var}\left(t_{m}\right)=\frac{t[1-t(1-\alpha)]}{N(1-\alpha)}
$$

La méthode de Green et al. (analyse multilocus) utilise une estimation du taux d'allofécondation $(t)$ basée sur le maximum du vraisemblance pour résoudre l'équation :

$$
\Sigma_{i}\left[\left(N_{i}-O_{i}\right) G_{i} /\left(1-G_{i} t\right)\right]-\Sigma_{i} O_{i} / t=0
$$

où $N_{i}$ est le nombre de descendants d'une mère $i, O_{i}$ est le nombre de descendants directement identifiés comme issus d'allofécondations (ils possèdent au moins un allèle absent chez la mère) et $G_{i}$ la probabilité de détecter un descendant issu d'allofécondation qui est égale au complément à 1 de la fréquence dans le pollen de la combinaison génotypique identique à celle produite par la mère.

Pour les deux méthodes utilisées, le pool pollinique parental a été assimilé soit à l'ensemble des individus de la parcelle (cette dernière étant représentative alors de l'ensemble du peuplement) soit au pool pollinique efficace ayant contribué effectivement à la constitution des descendants. Dans le cas de la deuxième méthode, on estime que les arbres mères peuvent recevoir préférentiellement du pollen d'autres arbres y compris ceux situés à l'extérieur de la parcelle.

Ont été également comparées entre elles, à l'aide d'un test d'homogénéité du $\chi^{2}$, les distributions alléliques dans le pollen ayant contribué à la formation des descendants et dans la population parentale (soit tous les individus de la parcelle dans ce cas).

\section{Résultats}

\section{A. Structure génétique de la station}

La distribution spatiale des génotypes pour chaque locus est illustrée par les figures 1,2 et 3 . Les fréquences alléliques et l'indice de fixation calculés à l'échelle de la parcelle sont représentés dans le tableau 1. L'indice de fixation, pour les trois locus (Fis $=0,006)$ n'est pas significativement différent de zéro au risque de $5 \%$ et indique une situation qui correspondrait à celle de la panmixie.

TABLEAU 1

Fréquences alléliques et indice de fixation (Fis) pour chacun des trois locus $P G I-I, I D H-I$ et $A D H-I$

Allele frequencies and fixation index (Fis) at each of the three loci, PGI-I, IDH-1 and ADH-I

\begin{tabular}{c|c|c|c}
\hline Locus & PGI-1 & IDH-1 & ADH-1 \\
\hline Allèles . . . . . . . & $1,001,201,090,800,76$ & $1,001,100,76$ & $1,001,160,76$ \\
\hline Fréquences alléliques & $0,740,010,080,120,05$ & $0,870,010,12$ & $0,940,010,05$ \\
\hline Fis $\ldots \ldots \ldots \ldots$ & $0, \ldots \ldots$ & 0,03 & $-0,05$ \\
\hline
\end{tabular}




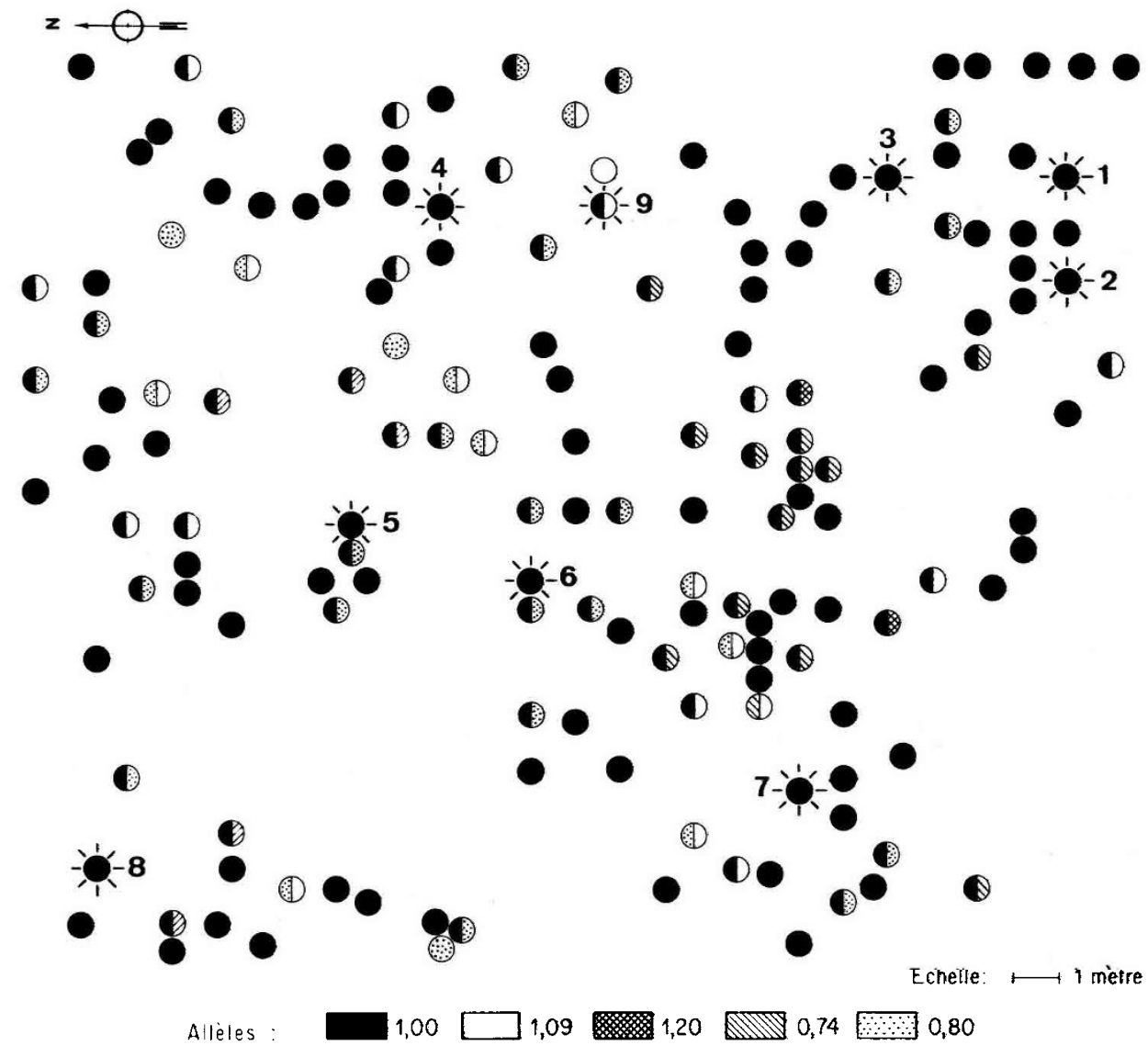

Fig. 1

Distribution spatiale des génotypes au locus PGI-1. Location of genotypes at the PGI-1 locus.

Individus à partir desquels ont été prélevés les descendants.

- Individuals from which progenies were collected. 


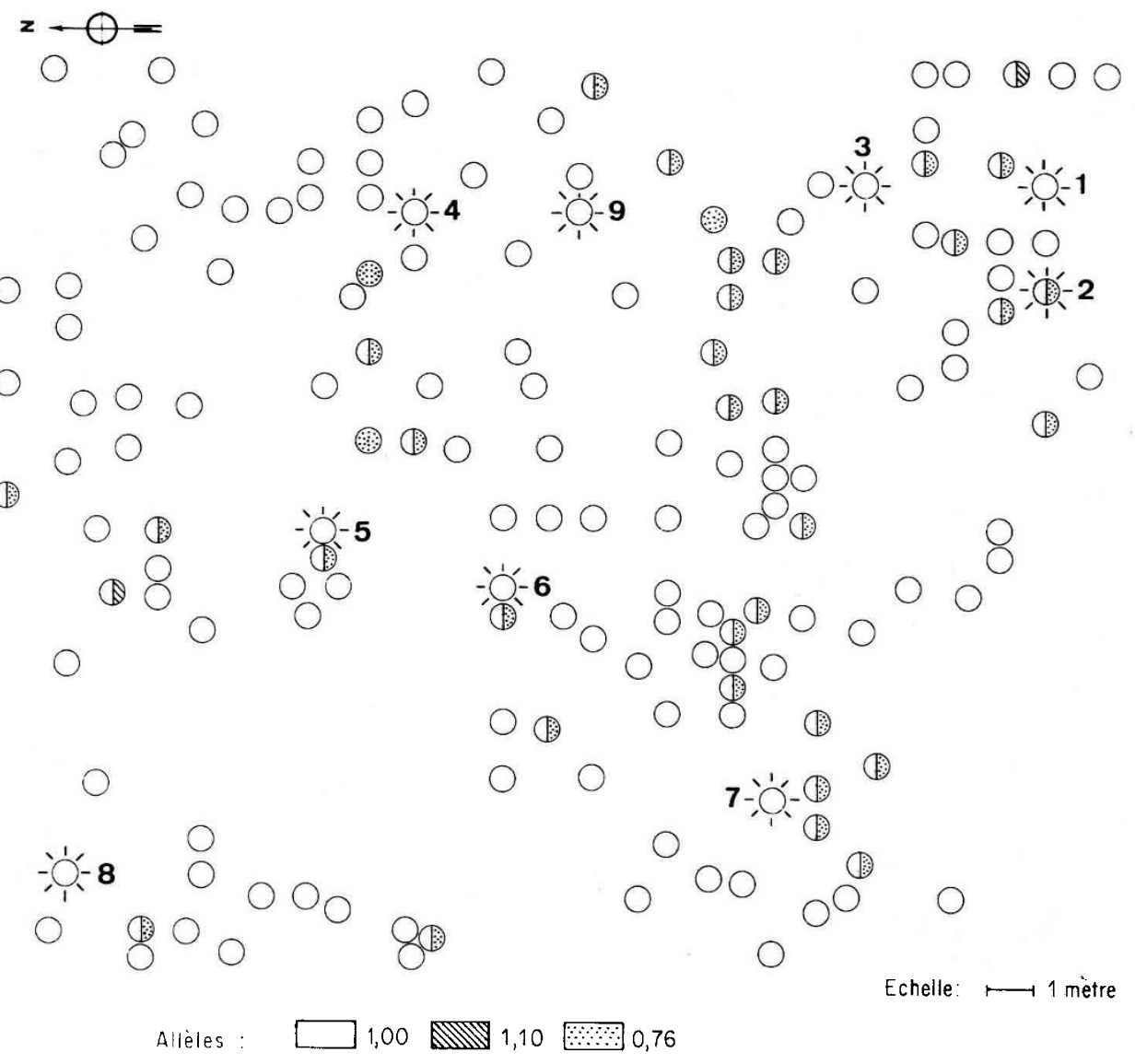

FiG. 2

Distribution spatiale des génotypes au locus IDH-I.

Location of genotypes at the IDH-I locus.

-' Individus à partir desquels ont été prélevés les descendants.

Individuals from which progenies were collected. 


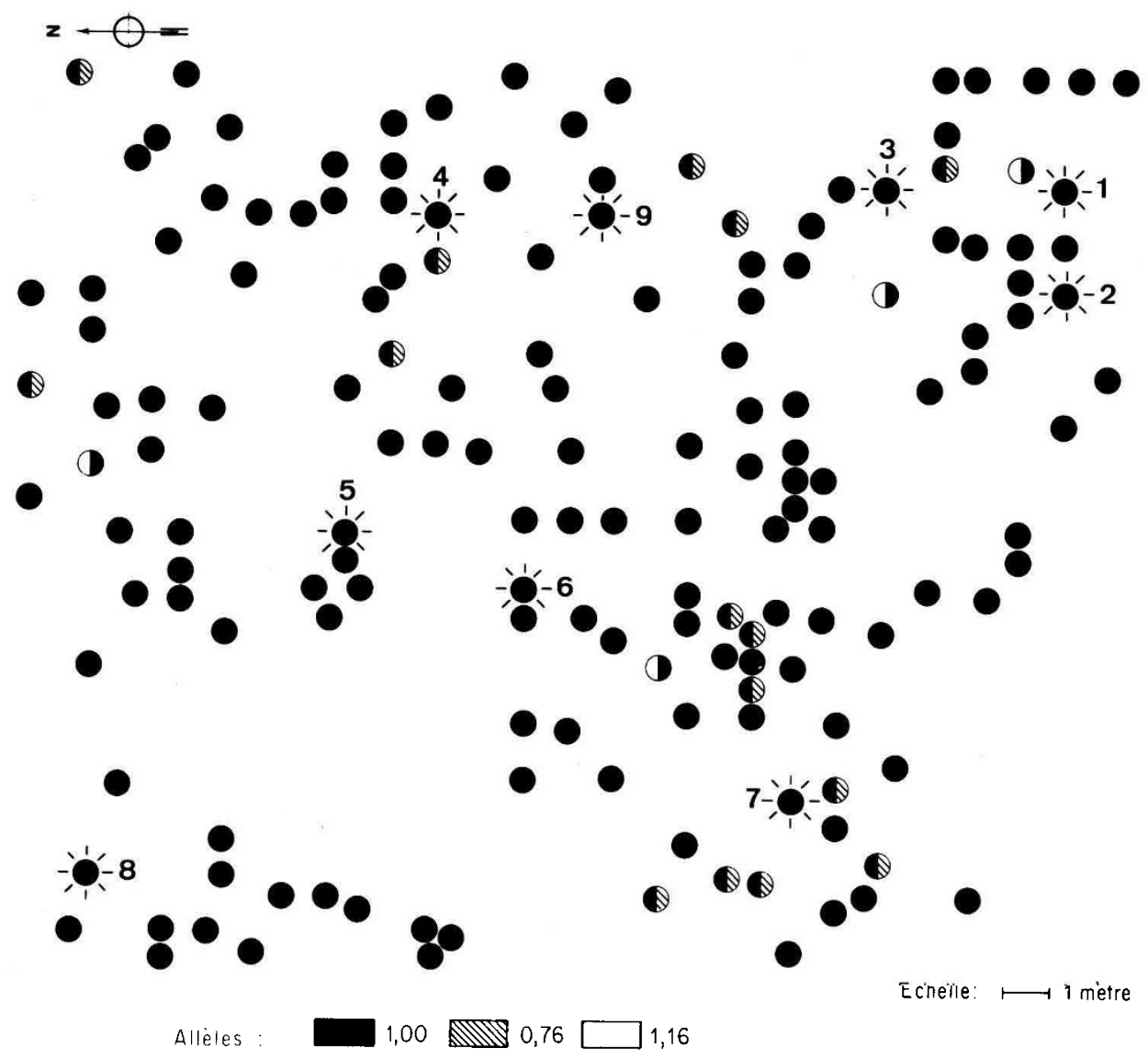

FIG. 3

Distribution spatiale des génotypes au locus $A D H-1$. Location of genotypes at the ADH-I locus.

- Individus à partir desquels ont été prélevés les descendants.

Individuals from which progenies were collected.

Les variations des indices Fis et Fst, pondérés en fonction du nombre d'individus par carré, à chaque échelle de subdivision, sont illustrées pour chaque locus, de la manière préconisée par WrIGHT (1978), par la figure 4. En abscisse, est reportée la taille des mailles représentée par la moitié du côté du carré transformé en son logarithme et en ordonnée sont indiqués les indices Fis et Fst. L'examen de ces trois figures montre une diminution progressive de Fst et une augmentation de Fis en fonction de la taille croissante des mailles.

Pour des valeurs de logarithmes népériens de tailles de mailles de 1,$4 ; 2,0 ; 2,3$; 2,$6 ; 2,8$ et 3,0 le nombre moyen d'individus par maille est respectivement de 1, 2, 6, 17,39 et 51 pour chaque locus. 
Pour le locus PGI-1, deux échelles de subdivisions correspondent à des situations d'homogénéité maximale des distributions d'effectifs entre mailles. Elles sont indiquées sur la figure 4 par les repères A et B. L'examen des indices Fis et Fst à chacune d'elles montre une absence de différenciation à l'intérieur des mailles et une très faible variation entre celles-ci. Pour le locus IDH-1, les valeurs de Fis et Fst, à l'échelle de la subdivision en 4 carrés (fig. 4), indiquent une tendance à la différenciation à l'intérieur des carrés et entre ceux-ci ; en effet, l'observation de la figure 1 montre une concentration relative des homozygotes pour l'allèle 1,00 et des hétérozygotes respectivement pour les allèles 1,00 et 0,76 dans les deux quarts supérieurs de la parcelle. Le locus ADH-1 présente d'ailleurs un très faible degré de polymorphisme, ce qui explique l'excès apparent d'hétérozygotes $(F i s<0)$ quelle que soit la taille de la maille considérée (fig. 4). L'indice $F s t$, à l'échelle de subdivision en quatre carrés, prend là encore une très faible valeur $(0,02)$ équivalente à celles obtenues pour les deux autres locus.

PGI - 1

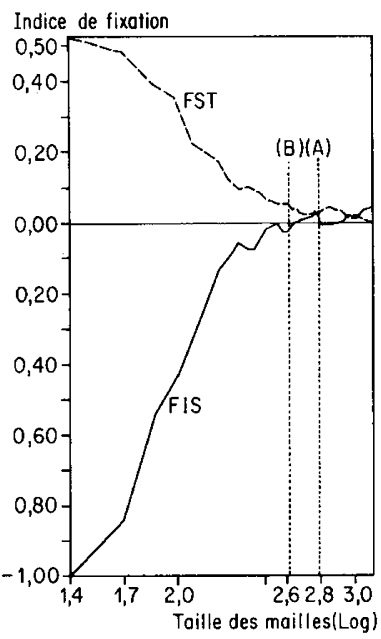

IDH - 1

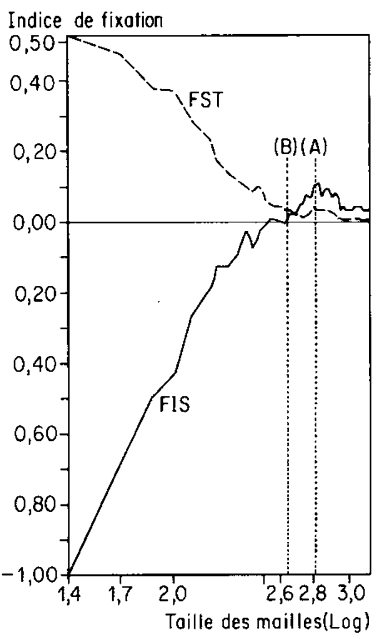

ADH - 1

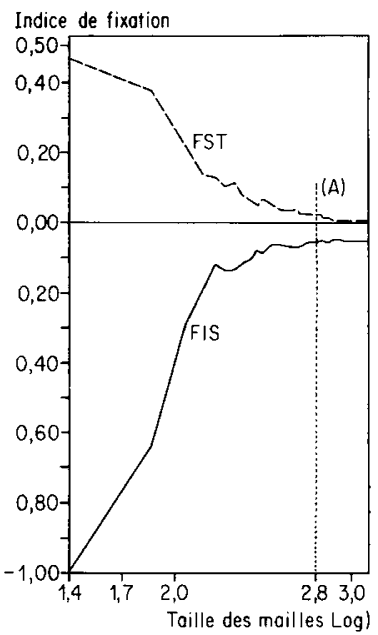

FIG. 4

Variation des indices Fis et Fst à chaque locus en fonction des diverses classes de subdivision. Les repères $(A)$ et $(B)$ correspondent à des échelles de subdivision pour lequelles les écarts-types des variations d'effectif ont des écarts-types $<6$ avec des effectifs par carré $>5$ :

$(A)$ : découpage en 9 carrés; $(B)$ : découpage en 4 carrés.

Fis and Fst variation at loci PGI-I,IDH-I and ADH-I respectively according to subdivided classes. $(A)$ and $(B)$ indicate the scale for which standard deviation between classes is $<6$ and the number of individuals per class is $>5$.

Si l'on se réfère aux travaux de WRIGHT (1978) portant sur Linanthus parryae, espère annuelle, principalement allogame, les valeurs des indices Fis et Fst sont toujours positives et leurs variations en fonction de plusieurs niveaux de taille croissante de subdivision forment respectivement une courbe croissante pour Fis et décroissante pour Fst . L'intersection des deux courbes correspond à la taille du voisinage. Selon 
WRIGHT, une telle variation est bien typique de celle attendue lorsque la différenciation à tous les niveaux considérés résulte d'un isolement par la distance. Il apparait donc clairement que l'on ne se situe pas dans le cas décrit par l'auteur, tout au moins à l'échelle de la parcelle.

\section{B. Analyse de la composition allélique des descendants}

\section{Comparaison des distributions alléliques dans le pollen ayant contribué à la formation des descendants}

La distribution des fréquences alléliques aux trois locus dans le pollen, autopollen (éventuel) et allopollen confondus, est illustrée par la figure 5 . Ne sont représentées que les descendances issues des 8 mères homozygotes pour l'allèle 1,00 des locus PGI-1 et IDH-1 et 9 mères homozygotes pour l'allèle 1,00 du locus $\mathrm{ADH}-1$. Une comparaison de ces distributions a été réalisée entre les descendances à l'aide du test d'homogénéité $\mathrm{du} \chi^{2}$. Les résultats obtenus sont présentés dans le tableau 2.

$\mathrm{Au}$ locus PGI-1, la distribution des fréquences alléliques dans le pollen de la descendance $\mathrm{n}^{\circ} 2$ est significativement différente au risque de $5 \%$ de celles des descendances $n^{\circ} 1,3$ et 6 . L'observation de la localisation de leur mère respective $\left(\mathrm{n}^{\mathrm{o}} 2,1,3\right.$ et 6 sur la figure 1) dans la station laisse apparaître que ces différences entre descendances ne résultent pas de la distance qui sépare les mères.

En effet, les mères 1 et 2 ne sont séparées entre elles que par un individu et elles sont, de par leur localisation en limite de parcelle, soumises aux mêmes effets de bordure et au même pool pollinique potentiel. L'allèle rare 1,20 n'est présent que dans trois descendances : 1,5 et 6 (fig. 5), alors que sa localisation dans la parcelle est pourtant plus proche des mères 7 et 3 . L'entourage immédiat de la mère $n^{\circ} 6$ est constitué d'arbres portant l'allèle 0,80 ; néanmoins, elle présente dans sa descendance une plus grande proportion de l'allèle 1,09 . On remarque là encore que les arbres ne sont pas préférentiellement pollinisés par ceux qui leur sont le plus proches.

$\mathrm{Au}$ locus IDH-1, les descendances $\mathrm{n}^{\circ} 3$ et 4 sont significativement différentes au risque de $5 \%$ des autres descendances par la distribution des fréquences alléliques dans le pollen ayant contribué à leur formation respective (tableau 2). La première ( $\mathrm{n}^{\mathrm{o}} 3$ ) est très peu polymorphe, la fréquence de l'allèle 0,76 est de $7 \%$, l'allèle rare $1,10 \mathrm{y}$ est absent ; la descendance $\mathrm{n}^{\circ} 4$ est, quant à elle, monomorphe pour l'allèle dominant 1,00 (fig. 4) ; l'allèle rare 1,10 n'est présent que dans deux descendances $\left(\mathrm{n}^{\circ} 1\right.$ et 6$)$; cet allèle se trouve à deux endroits dans la parcelle (fig. 2), à proximité de la mère $\mathbf{n}^{\mathrm{o}} 1$; on pourrait alors supposer que l'arbre portant cet allèle l'aurait effectivement pollinisée. La mère $n^{\circ} 6$ se trouve relativement éloignée des deux arbres portant cet allèle et plusieurs individus l'en séparent. Il apparaît au vu de ces résultats que la distance entre les arbres n'intervient pas de façon systématique dans leur pollinisation.

$\mathrm{Au}$ locus $\mathrm{ADH}-1$, les diverses descendances ne présentent entre elles aucune différence dans la distribution des fréquences alléliques du pollen ayant contribué à leur formation, ceci étant vraisemblablement dû au faible degré de polymorphisme à ce locus. Notons néanmoins que l'allèle rare 1,16 n'est présent que dans trois descendances : $\mathrm{n}^{\circ} 5,6$ et 9 (fig. 5) et que la descendance $n^{\circ} 1$ est monomorphe (elle ne présente que l'allèle le plus fréquent 1,00 ). 

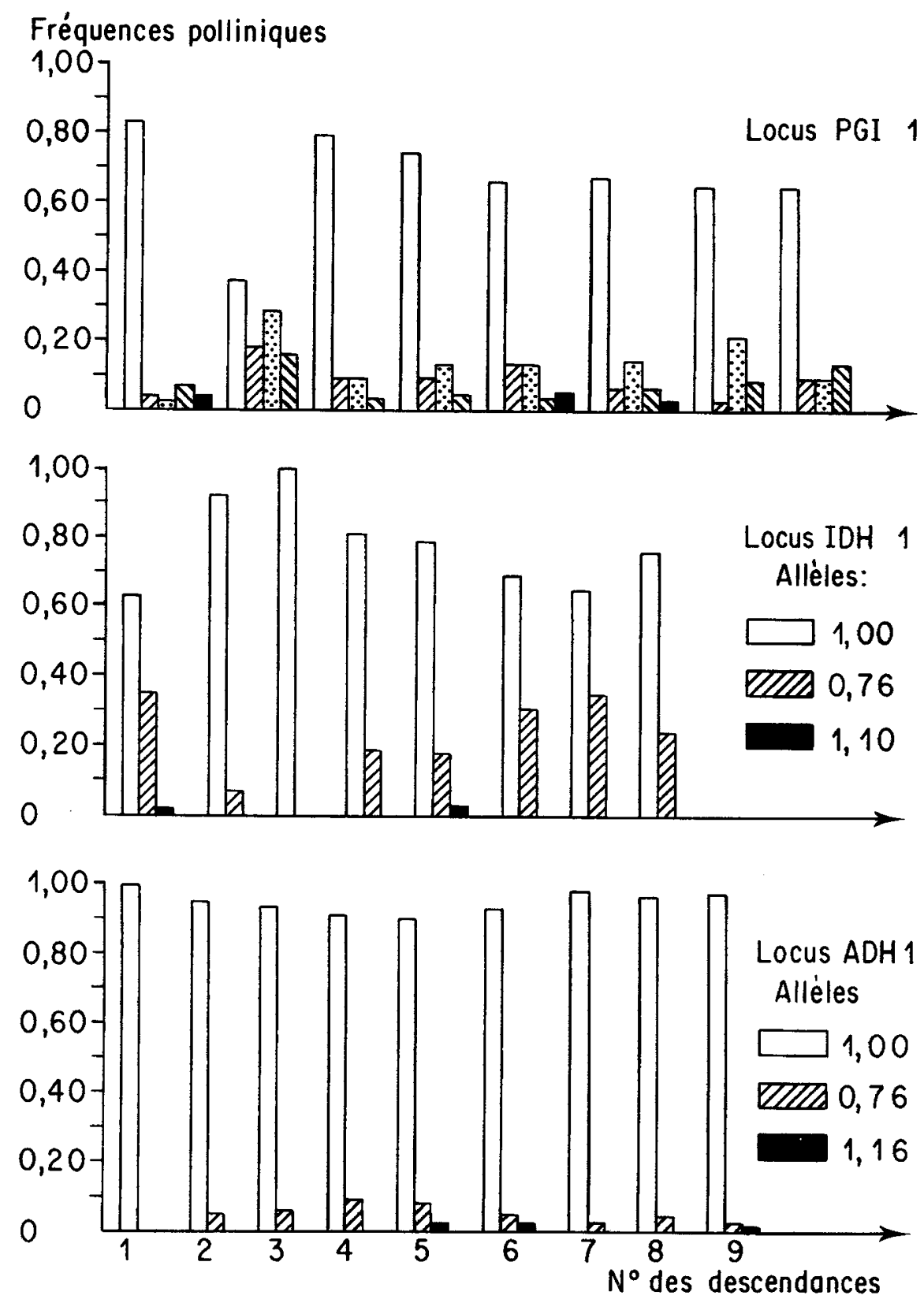

Allèles: $\square$ 1,00 WIIA 0,74 1,09

FIG. 5

Distribution des fréquences alléliques dans le pollen ayant contribué à la formation des descendants. Allele frequencies distribution in the effective pollen responsible for the observed progenies. 
TABLEAU 2

Comparaison entre descendances des distributions alléliques aux locus PGI-I et IDH-I : valeurs obtenues à partir du test d'homogénéité $d u \chi^{2}$

Comparison between the progenies of allele distributions at loci PGI-I and IDH-I : data obtained from $\chi^{2}$

\begin{tabular}{|c|c|c|c|c|c|c|c|c|c|}
\hline $\begin{array}{l}\mathbf{N}^{\circ} \text { de } \\
\text { descen- } \\
\text { dances }\end{array}$ & 1 & 2 & 3 & 4 & 5 & 6 & 7 & 8 & \multirow{10}{*}{ PGI-1 } \\
\hline 1 & $\infty$ & $* 13,56$ & & & & & & & \\
\hline 2 & & & $* 8,53$ & & & $* 7,77$ & & & \\
\hline 3 & & & $\infty$ & & & & & & \\
\hline 4 & $* 10,66$ & & $* 9,24$ & $\approx$ & & & & & \\
\hline 5 & & & & & & & & & \\
\hline 6 & & & & & & & & & \\
\hline 7 & & & $* 9,05$ & $* 9,29$ & & . & & & \\
\hline 8 & & & $* 7,69$ & $* 9,60$ & & & & & \\
\hline 9 & & & $* 9,07$ & $* 9,46$ & & & & & \\
\hline
\end{tabular}

IDH-1

${ }^{*} P<0,05$.

WIDEescendance issue de la mère hétérozygote au locus IDH-1, non incluse dans les comparaisons pour ce locus. Progeny from a parent tree heterozygote at locus IDH-1. This was not included in comparisons involving the locus.

\section{Estimation du taux d'allofécondation}

Le taux d'allofécondation a été estimé pour chaque mère suivant la méthode de SHAw et al. (1981) qui utilise plusieurs locus à la fois; ces auteurs évaluent en premier lieu la probabilité $(\alpha)$ de ne pas détecter une allofécondation alors qu'elle a eu lieu, cette probabilité étant ensuite utilisée pour le calcul du taux d'allofécondation. Cette méthode requiert pour son application directe la présence, au niveau des mères, de toutes les combinaisons génotypiques, ce qui n'est pas le cas dans la présente étude. De ce fait, des modifications ont été apportées pour l'estimation de $\alpha$. Deux séries de calcul ont été réalisées. Dans la première série, la probabilité $\alpha$, a été calculée à partir d'un pool pollinique identique à la composition allélique de la station, ce qui suppose que les descendants résultent d'un régime panmictique. La deuxième série d'estimations $\left(\alpha_{2}\right)$ utilise le pool pollinique ayant contribué à la formation des descendants, autopollen (éventuel) et allopollen confondus. L'estimation globale du taux d'allofécondation a été réalisée à partir de la méthode de GrEen et collaborateurs (1980) avec les mêmes modifications que pour la méthode précédente.

Les résultats obtenus pour les estimations par mère (tableau 3) montrent, dans le cas de la première méthode, une variation du taux d'allofécondation $t_{l}$ entre mères : en effet, deux d'entre elles $\left(\mathrm{n}^{\circ} 3\right.$ et 4 ) présentent des taux inférieurs à 1 , respectivement 0,63 et 0,88 alors que dans la deuxième série d'estimation $t_{2}$, toutes les mères se sont 
avérées strictement allogames. Afin de vérifier l'hypothèse de la panmixie et donc, la validité de la première série de résultats, une comparaison des fréquences polliniques a été effectuée entre l'ensemble des descendants et la population parentale. L'estimation globale du taux d'allofécondation par la méthode de Green et al. (1980) donne, pour les deux séries d'estimation, un taux supérieur à 1. Dans le premier cas (situation panmictique), $t$ est égal à 1,2 et dans le deuxième cas, $t$ est égal à 1,3 . Les valeurs de $t$ supérieures à 1 traduisent un excès d'hétérozygotes (donc, dans ce cas, d'individus issus d'allofécondations) et un déficit d'homozygotes pour les allèles possédés par les arbres mères, par rapport aux fréquences attendues à partir du pool allélique de la parcelle (série 1), ou même des fréquences alléliques dans le pollen ayant contribué à la formation des descendants (série 2). Cet excès d'hétérozygotes serait attribuable à des pollinisations préférentielles par des arbres possédant des allèles distincts de ceux des arbres mères.

\section{TABleau 3}

Estimation du taux d'allofécondation pour chaque mère, selon la méthode de $S_{H A W}$ et al. (1981) Outcrossing rate estimation for each parent tree according to SHAW's method (SHAW et al., 1981)

\begin{tabular}{c|c|c|c|c|c|c|c|c|c}
\hline \hline \multirow{2}{*}{$\begin{array}{c}\mathrm{N}^{\circ} \text { de } \\
\text { la mère }\end{array}$} & \multicolumn{3}{|c|}{$\begin{array}{c}\text { Génotypes de } \\
\text { la mère }\end{array}$} & \multirow{2}{*}{$N_{i}$} & $O_{i}$ & $\alpha_{l}$ & $t_{l}$ & $\alpha_{2}$ & $t_{2}$ \\
\cline { 2 - 9 } & PGI-1 & IDH-1 & ADH-1 & & & & & & \\
\hline 1 & $A A$ & $A A$ & $A A$ & 44 & 21 & 0,69 & 1,50 & 0,66 & 1,4 \\
\hline 2 & $A A$ & $A B$ & $A A$ & 38 & 22 & 0,79 & 2,70 & 0,62 & 1,5 \\
\hline 3 & $A A$ & $A A$ & $A A$ & 33 & 10 & 0,49 & 0,63 & 0,73 & 1,2 \\
\hline 3 & $A A$ & $A A$ & $A A$ & 23 & 7 & 0,61 & 0,88 & 0,75 & 1,2 \\
\hline 5 & $A A$ & $A A$ & $A A$ & 63 & 33 & 0,71 & 1,80 & 0,61 & 1,3 \\
\hline 6 & $A A$ & $A A$ & $A A$ & 133 & 70 & 0,52 & 1,10 & 0,62 & 1,4 \\
\hline 7 & $A A$ & $A A$ & $A A$ & 51 & 27 & 0,71 & 1,80 & 0,61 & 1,3 \\
\hline 8 & $A A$ & $A A$ & $A A$ & 23 & 12 & 0,64 & 1,40 & 0,58 & 1,2 \\
\hline 9 & $A B$ & $A A$ & $A A$ & 82 & 32 & 0,74 & 1,50 & 0,67 & 1,2 \\
\hline
\end{tabular}

Dans la représentation des génotypes, $A$ correspond à l'allèle le plus fréquent, soit 1,00 pour les trois locus, la lettre $B$ correspond à l'allèle 0,76 du locus IDH-1 et à l'allèle 1,09 du locus PGI-1.

In genotypes, A correspond to the most frequent allele, B correspond to either IDH-1 0.76 or PGI-1 1.09.

$N_{i}$ : Effectif des descendants par mère.

Number of individuals in each progeny.

$O_{i}$ : Nombre de descendants issus d'allofécondation directement décelables.

The number of detectable outcrosses progenies.

$\alpha_{l}$ : Probabilité de ne pas détecter une allofécondation alors qu'elle a eu lieu, calculée à partir d'un pool pollinique supposé identique à la composition de la station.

Probability of not detecting an outcross when it has occurred, assuming that the pollen pool is given by that of the entire site.

$t_{l}$ : Taux d'allofécondation calculé à partir de $\beta_{\text {I }}$

Outcrossing rate, calculated using $\alpha_{1}$.

$\alpha_{2}$ : Probabilité calculée à partir du pool pollinique ayant participé à la formation des descendants (autopollen éventuel et allopollen confondus).

Probability of not detecting an outcross when it has occurred assuming that the pollen pool is given by those alleles having formed offspring (self and outcrossed pollen are confounded).

$t_{2}$ : Taux d'allofécondation calculé à partir de $\alpha_{2}$.

Outcrossing rate, calculated using $\alpha_{2}$. 
La distribution des fréquences alléliques dans le pollen ayant contribué à la formation des descendants et dans la population parentale se sont avérées significativement différentes au risque de $5 \%$ pour les locus PGI-1 et IDH-1. Ces résultats infirment l'hypothèse de la panmixie. En conséquence, la deuxième série d'estimation du taux d'allofécondation concorderait avec les résultats expérimentaux précédemment cités. Il apparaît, au regard de l'ensemble de ces données que, d'une part, le régime de reproduction n'est pas panmictique et que, d'autre part, les variations entre descendances ne seraient pas dues à des différences du taux d'allofécondation entre les mères. Bussma et al. (1986) expliquent ces variations pour l'espèce Zea mays par une hétérogénéité temporelle du pool pollinique; cette dernière résulterait, selon ces mêmes auteurs, du décalage phénologique entre les individus de la population étudiée. Une telle explication serait appropriée au cas du chêne vert qui présente un étalement relativement important du temps de floraison au sein d'une même station (DU MERLE, 1983).

\section{Discussion}

Les résultats obtenus à partir de l'étude de la structure génétique d'une population de chêne vert, abordée ici par l'analyse de l'organisation spatiale des génotypes de l'ensemble des arbres constituant $490 \mathrm{~m}^{2}$ de cépée, mettent en évidence d'une part une situation proche de l'équilibre de Hardy-Weinberg et d'autre par l'absence d'un effet d'agrégation spatiale des diverses classes génotypiques aux trois locus considérés.

En ne prenant en compte que cette première série de résultats, plusieurs interprétations sont possibles. 1) En supposant que l'espèce fonctionne suivant un modèle d'isolement par la distance et en considérant d'une manière concomitante que quelques générations au moins se seraient écoulées, il faudrait admettre que la station qui rassemble la totalité des individus étudiés correspondrait à la taille du voisinage ou lui serait inférieure. 2) Une autre possibilité est que la population étudiée soit jeune : peu de générations, chevauchantes ou non, se seraient écoulées depuis sa fondation, de sorte qu'aucune structuration n'aurait eu le temps de s'établir. Comme cela a déjà été énoncé ci-dessus, cette situation ne nous paraît pas improbable si l'on se réfère à la fois aux caractéristiques propres à l'espèce (longévité très importante, très peu de régénérations par les glands), aux traitements de coupes (environ tous les 30 ans) auxquels la région où se trouve la cépée étudiée est soumise (les rejets de souche augmentent la longévité des arbres) et aux données de l'histoire régionale qui feraient remonter le dernier grand défrichement de la garrigue montpelliéraine et donc le renouvellement des individus, à la fin du $\mathrm{XVIII}^{\mathrm{c}}$ siècle (Dugrand, 1964). Compte tenu de la faible profondeur du sol à de nombreux endroits, les superficies continues défrichées pour les cultures n'étaient jamais très étendues et l'allogamie du chêne vert associé à sa variabilité phénologique infrapopulationnelle (voir ci-dessus) ont certainement contribué à limiter considérablement les effets de fondations et de dérive génétique au moment de la recolonisation suivant l'abandon des cultures.

L'analyse des distributions alléliques dans les descendances d'un certain nombre d'individus, localisés dans cette même station, indique :

- que le chêne vert est une espèce allogame quasiment stricte : ce résultat est déduit des estimations des taux d'allofécondation et des résultats expérimentaux concer- 
nant des autofécondations forcées (données non publiées). Les périodes de maturité des fleurs mâles et femelles étant le plus souvent synchrones sur un même arbre, on pourrait envisager l'existence d'un système d'auto-incompatibilité ; des études génétiques adéquates seraient nécessaires pour le démontrer ;

- qu'il existe une grande hétérogénéité du pollen participant à la fécondation des différents arbres, ce qui pourrait correspondre à un régime de reproduction non panmictique. La possibilité de variation du taux d'allofécondation entre mères, souvent invoquée pour expliquer un tel résultat chez de nombreuses espèces dont Eucalyptus obliqua (BRown et al., 1975), Clarkia exilis (VASEK, 1967), Elymus canadensis (SANDERS \& Hamrick, 1980 ; VASEK, 1967) et Elymus canadensis (SANDER \& Hamrick, 1980) ne peut être prise en compte dans le cas du chêne vert à cause des résultats expérimentaux (YACINE, 1987, non publié) qui convergent dans le sens d'une allogamie stricte de l'espèce. Par contre, l'hétérogénéité du flux pollinique est d'origine temporelle (décalage phénologique important, indépendant semble-t-il de la localisation des arbres de la station). De la sorte, les arbres précoces ne peuvent se croiser avec les arbres tardifs.

Cette hétérogénéité se manifeste aussi par la capacité de l'espèce à présenter une variabilité individuelle considérable quant à l'investissement des arbres dans les fonctions végétatives et reproductrices. Parmi ces dernières, on peut citer les fonctions mâles et femelles (données non publiées). Ainsi, les individus ne sont pas nécessairement pollinisés par leurs voisins immédiats mais par ceux (pas trop éloignés) qui leur sont à la fois phénologiquement synchrones et qui auront prioritairement investi dans la fonction mâle.

Les processus qui interviennent dans le régime de reproduction de l'espèce ont des effets qui s'opposent. Ainsi, l'allogamie stricte a pour conséquence de limiter l'effet de consanguinité, alors que la variabilité dans la phénologie et dans l'investissement des fonctions mâles et femelles a pour conséquence une réduction de l'effectif génétique de reproduction et une diminution de la production de glands. Dans les cas où l'on considère des générations successives, l'impact de ce mode de fonctionnement sur la structuration génétique d'une population peut être envisagé de plusieurs manières :

1) une composition identique des groupes de floraison au cours des années et une stabilité des arbres quant à leur mode d'investissement dans les fonctions végétatives et reproductrices (mâles et femelles) d'une année sur l'autre, devraient aboutir à une structuration spatiale, après quelques générations seulement ;

2) une variation interannuelle, à la fois de la composition des groupes de floraison et du mode d'investissement dans les fonctions mâles et femelles devrait au contraire freiner la structuration spatiale et maintenir une diversité génétique relativement importante au sein de la population. Cette deuxième hypothèse permettrait, mieux que la première, d'expliquer la situation observée dans la population de Puéchabon si l'on admettait qu'un nombre non négligeable de générations se soient écoulées (ce qui n'est pas l'hypothèse présentée comme étant la plus plausible). En réalité, les études dont on peut disposer portant sur la reproductibilité des caractéristiques individuelles de la phénologie du chêne vert sont encore très sporadiques. Elles n'ont pas été prolongées suffisamment longtemps pour permettre de trancher entre les deux hypothèses. Les premières informations mettent en évidence à la fois une certaine répétabilité des observations mais aussi des cas signalés de changements radicaux, sans qu'il soit possible, pour l'instant, d'apprécier la part de l'un et de l'autre de ces effets. Pour d'autres espèces, telles que Pseudotsuga menziesii (EL KASSABy et al., 1984), il apparaît que les groupes de floraison se maintiennent d'une année sur l'autre, mais à notre connaissance, l'impact de ce paramètre sur la structure génétique des populations n'a 
pas été étudié. La comparaison de la structure génétique dans une population en place et dans sa descendance a porté sur des espèces qui ne présentent pas l'ensemble des caractères spécifiques au chêne vert. Dans la plupart de ces cas, l'hétérogénéité du pool pollinique observé résulte d'une variation du taux d'allofécondation. C'est le cas d'Ipomea purpurea (ENNOS \& CLEGG, 1982).

Si l'on admet que très peu de générations ont contribué à la constitution des populations telles que celle de la parcelle étudiée, la reproductibilité de la phénologie des individus n'a plus guère d'impact sur la structure de la population et n'est donc plus à prendre en compte. Dans ce cas, la signification adaptative des caractéristiques particulières du régime de reproduction du chêne vert, notamment celle touchant à la variation phénologique individuelle, pourrait être la suivante : lors de la constitution de nouvelles populations, consécutivement à un bouleversement profond de l'environnement, comme par exemple un défrichement intense ou bien un feu très sévère, un nombre réduit d'individus de chêne vert, participent, par leur descendance, à la recolonisation du milieu. Une diversité génétique spatiale importante est rapidement reconstituée uniquement grâce à la variabilité phénologique. Celle-ci a pour effet de diversifier au maximum les arbres producteurs de pollen contribuant à la fécondation des individus producteurs de glands. Elle évite ainsi au maximum les effets de consanguinité (principalement entre les arbres proches) auxquels une espèce allogame peut être sensible. En tout état de cause, le chêne vert, par son allogamie stricte, sa variabilité pour la phénologie et dans l'investissement des fonctions mâles et femelles, s'avère être une espèce originale et constituerait un modèle particulièrement intéressant pour l'étude des processus microévolutifs.

Reçu le 20 mars 1987.

Accepté le 6 octobre 1987.

\section{Remerciements}

Nous tenons tout particulièrement à remercier Messieurs C. Gliddon, P.H. Gouyon et J.D. LEBRETon qui ont réalisé pour nous les programmes pour le traitement des données. Nous remercions également Monsieur F. Romane qui a régulièrement participé au travail de terrain ainsi que Madame Panis pour sa collaboration technique.

\section{Références bibliographiques}

Aissa D., 1981. Etude expérimentale de la germination du chêne vert (Quercus ilex L.). Thèse de spécialité, Univ. Sci. Tech. Saint-Jérôme, Marseille.

Baradat P., Marpeau A., Bernard-Dagan C., 1984. Les terpènes du pin maritime, aspects biologiques et génétiques. 6 . Estimation du taux moyen d'autofécondation et mise en évidence d'écarts à la panmixie dans un verger à graines de semis. Ann. Sci. For., 41, 107-134.

Bijlsma R., Allard R.W., Kahler A.L., 1986. Non random mating in a open pollinated maize population. Genetics, 112, 669-680.

Brown A.H.D., Matheson A.C., Eldridge K.G., 1975. Estimation of the mating system of Eucalyptus obliqua, L'Herit. by using allozyme polymorphisms. Aust. J. Bot., 22, 931-949.

Brunel D., Rodolphe F., 1985. Genetic neighbourhood structure in a population of Picea abies L. Theor. Genet., 71, 101-110. 
Cheliak M.W., Dancik B.P., Morgan J., Yeh F.C.H., Strobeck C., 1985. Temporal variation of the mating system in a natural population of Jack pine. Genetics, 109, 569-584.

Dugrand R., 1964. La garrigue montpelliéraine : essai d'explication d'un paysage. 292 p., Presses Universitaires de France, Paris.

Du Merle P., 1983. Physiologies comparées du chêne pubescent, du chêne vert et de Tortrix viridana L. (Lep, Tortricidae). Mise en évidence chez l'insecte de deux populations sympatriques adaptées chacune à l'un des chênes. Acta Oecol., 4, 55-74.

El Kassaby Y.A., Fashler A.M.K., Sziklai O., 1984. Reproductive phenologie and its impact on genetically improved seed production in a Douglas-Fir Seed orchard. Silvae Genet., 33, 120126.

Ellstrand N.C., Foster K.W., 1983. Impact of population structure on the apparent outcrossing rate of grain sorghum (Sorghum bicolor). Theor. Appl. Genet., 66, 323-327.

EnNos R.A., 1985. The mating system and genetic structure in a perennial grass, Cynosurus cristatus L. Heredity, 55, 121-126.

Ennos R.A., Clegg M.T., 1982. Effect of population substructuring on estimates of outcrossing rate in plant population. Heredity, 48, 283-292.

Green A.G., Brown A.H.D., Oram R.N., 1980. Determination of outcrossing rate in a breeding population of Lupinus albus L. (white lupin). Z. Pflanzenzücht., 84, 181-191.

Kirby G.C., 1975. Heterozygote frequencies in small subpopulation. Theor. Pop. Biol., 8, 31-48.

McNeilly T., ANtonovics J., 1968. Evolution in closely adjacent plant population. 4. Barriers to gene flow. Heredity, 23, 205-218.

NeI M., 1977. F-statistics and analysis of gene diversity in subdivided population. Ann. Hum. Genet., 41, 225-233.

Roberds J.H., ConKLE M.T., 1984. Genetic structure in loblolly pine stands allozyme variation in parents and progeny. For. Sci., 30, 319-329.

Sander T.B., Hamrick J.L., 1980. Variation in the breeding system of Elymus canadensis. Evolution, 34, 117-122.

Shaw D.V., Kahler A.L., Allard R.W., 1981. A multilocus estimator of mating system parameters in populations. Proc. Natl. Acad. Sci. USA, 78, 1298-1302.

Stam P., 1983. The evolution of reproductive isolation in closely adjacent plant populations through differential flowering time. Heredity, 50, 105-118.

VASEK F.C., 1967. Outcrossing in natural populations. 3. The deer creek population of Clarkia exilis. Evolution, 21, 241-248.

Wright S., 1951. The genetical structure of populations. Ann. Eugen., 15, 323-354.

WRIGHT S., 1978. Evolution and the genetics of populations, vol. 4, Variability within and among populations. 580 p., University of Chicago Press, Chicago.

YACINE A., 1987. Une étude d'organisation de la diversité génétique inter et intra-population chez le chêne vert: Quercus ilex $\mathrm{L}$. Thèse de $3^{\mathrm{e}}$ cycle. Univ. Sci. Tech. Languedoc, Montpellier.

Yacine A., Lumaret R., 1988. Organization of genetic diversity in holm oak (Quercus ilex L.) insight from several allozyme markers. Silvae Genet., (à paraître). 